\title{
Role of Upper Respiratory Microbiota and Virome in Childhood Rhinitis and Wheeze: Collegium Internationale Allergologicum Update 2021
}

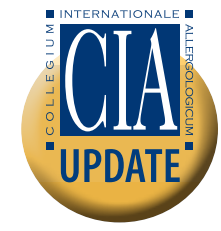

\author{
Carina Jing Xuan Tay ${ }^{a}$ Le Duc Huy Ta ${ }^{a}$ Yu Xiang Ow Yeong ${ }^{a} \quad$ Gaik Chin Yap ${ }^{a}$ \\ Justin Jang Hann Chu ${ }^{b, c}$ Bee Wah Lee ${ }^{a}$ Elizabeth Huiwen Tham ${ }^{a, d, e}$ \\ aDepartment of Paediatrics, Yong Loo Lin School of Medicine, National University of Singapore, Singapore, \\ Singapore; ${ }^{b}$ Department of Microbiology and Immunology, Infectious Diseases Translational Research Programme, \\ Yong Loo Lin School of Medicine, National University Health System, National University of Singapore, Singapore, \\ Singapore; 'Institute of Molecular and Cell Biology, Agency for Science, Technology and Research, Singapore, \\ Singapore; 'Khoo Teck Puat- National University Children's Medical Institute, National University Health System, \\ Singapore, Singapore; eHuman Potential Translational Research Programme, Yong Loo Lin School of Medicine, \\ National University of Singapore, Singapore, Singapore
}

\section{Keywords}

Microbiota $\cdot$ Virome $\cdot$ Nasal microbiome $\cdot$ Wheeze $\cdot$ Rhinitis

\begin{abstract}
There is emerging evidence that the respiratory microbiota influences airway health, and there has been intense research interest in its role in respiratory infections and allergic airway disorders. This review aims to summarize current knowledge of nasal microbiome and virome and their associations with childhood rhinitis and wheeze. The healthy infant nasal microbiome is dominated by Corynebacteriaceae and Staphylococcaceae. In contrast, infants who subsequently develop respiratory disorders are depleted of these microbes and are instead enriched with Proteobacteria spp. Although human rhinovirus and human respiratory syncytial virus are welldocumented major viral pathogens that trigger rhinitis and wheezing disorders in infants, recent limited data indicate that bacteriophages may have a role in respiratory health. Future work investigating the interplay between commensal microbiota, virome, and host immunological responses is an important step toward understanding the dynamics of the nasal community in order to develop a strategical approach to combat these common childhood respiratory disorders.
\end{abstract}

(c) 2021 S. Karger AG, Basel

\section{Introduction}

In recent years, the influence of commensal microbiota on the development of host immunity has been the focus of intense research. The commensal microbiota also maintains the integrity of mucosal and epithelial barriers and modulates an equilibrium between commensal and pathogen colonization, which in turn impacts upon health and disease states [1]. This, therefore, gives rise to the notion that establishing a symbiotic relationship between host and microbiota in early life is important and could influence health in later life [2]. In this regard, the influence of the gut microbiome on immunity has been the main focus of attention. However, emerging data have also provided evidence that the respiratory microbiota is associated with airway health and may affect the development of respiratory infections and disorders [3].

This review aims to present the current knowledge on the upper airway microbiota and its development through infancy and its influence on childhood respiratory infections and disorders such as rhinitis and wheeze. In view of the limited published work, we also intend to identify

Bee Wah Lee and Elizabeth Huiwen Tham are co-senior authors. Edited by: D.Y. Wang, Singapore.
Elizabeth Huiwen Tham, Division of Allergy, Immunology and Rheumatology Department of Paediatrics, Khoo Teck Puat - National University Children's Medical Institute, National University Health System of Singapore, Level 12 NUHS Tower Block 1E Kent Ridge Road, Singapore 119228 (Singapore)

elizabeth_tham@nuhs.edu.sg 
knowledge gaps and directions for future research. We also recognized that data comparisons between studies may be challenging as nasal sampling techniques (anterior nares vs. nasopharyngeal swabs) and molecular detection techniques varied considerably between studies. These factors have to be considered when interpreting data between studies.

\section{Methodology}

The literature search for this review, which included relevant cohort and case-control studies, was conducted between August and October 2020 using the Google Scholar and PubMed database. The search terms used were (1) ([nasal] OR [nose] OR [nasopharyngeal]) AND ([infant] OR [neonate]) AND ([rhinitis] OR [wheeze]) and (2) (nasal) OR (microbiome) OR (virome) OR (bacteriophage). The search was limited to English publications in the last 2 decades and children from birth to 18 years.

\section{Early Establishment and Succession of Nasal Microbiota in Infants}

The upper respiratory tract has been recognized as the main entry point [4] and reservoir [5] for respiratory commensal bacteria and pathogens. In adults, the bacterial composition of the nasal microbiome is stable and diverse as a result of maturation due to age progression and interaction with the environmental microbiome [6, 7]. In comparison, the nasal microbiome of children, especially under 3 years of age, demonstrates significant variability in composition, both between individuals and across age groups $[8,9]$. The data from these studies are presented as relative abundance, and data on the bacteria density are not available with the $16 \mathrm{~S}$ ribosomal RNA (rRNA) sequencing methods. Table 1 summarizes 5 studies performed on birth cohorts with repeated nasal sampling over first 1-2 years of life. Although general comparisons in nasal microbiome profiles can be made, it is recognized that there are some methodological differences between these studies. One such difference is the nasal sampling site. Two studies $[10,11]$ of the 5 studies sampled the anterior nares, whilst the other 3 utilized nasopharyngeal swabs $[9,12,13]$. It has been shown that the nasopharynx is more likely to be colonized by nonlipophilic skin colonizers such as Haemophilus spp. and Streptococcus spp.; thus, these species might be more likely to be enriched in studies using nasopharyngeal samples [11, $12,14,15]$. In terms of DNA sequencing techniques, 4 studies [9-12] utilized the $16 \mathrm{~S}$ rRNA 454 pyrosequencing technique, except for one [13] which applied the more advanced DNA shotgun sequencing techniques of the $16 \mathrm{~S}$ rRNA gene. These techniques support comparisons for larger-order bacterial taxa. However, the low sequencing depth precludes species-specific comparisons [16].

The overall results of these studies show that nasal microbiome undergoes dynamic changes in microbiota composition throughout the first few years of life. There is low microbial diversity soon after birth, and this increases over the next few months of life. Five longitudinal studies from European and American birth cohorts involving nasal sampling over the first 2 years of life showed that the nasopharyngeal microbiome of healthy infants is dominated by a high abundance of Corynebacteriaceae (Actinobacteria phylum), Moraxellaceae (Proteobacteria phylum), and Staphylococcaceae (Firmicutes phylum), with some studies describing also the presence of Pasteurellaceae (Proteobacteria phylum), Propionibacteriaceae (Actinobacteria phylum), and Lactobacillaceae (Firmicutes phylum) in the first few months of life. These early colonizers are subsequently replaced by a dominance of Streptococcaceae (Firmicutes phylum) and/or either Corynebacteriaceae or Moraxellaceae and other minor families [9-13]. Based on these studies, the changing profile of the nasal microbiome in the first 2 years of life is depicted in Figure 1. The adolescent/adult nasal microbiome profile is also included as a comparison $[17,18]$. The study from the Netherlands and Switzerland demonstrated strong seasonal variability of microbial profiles $[9,11]$. The winter profile was dominated by Pasteurellaceae (Proteobacteria phylum), while the summer was dominated by Corynebacteriaceae (Actinobacteria phylum) and/or Bacteroidetes phylum. The differences were postulated to be a result of interactions between seasonal infection patterns and the host-environment equilibrium, where disturbances to the native nasal microbiota could be induced by infection by pathogenic bacteria in the environment.

The consistent presence and dominance of the commensals Corynebacteriaceae and Staphylococcaceae in the nasal microbial profiles in early life have been substantiated by the longitudinal nasal microbial profiles of healthy controls from our study [19]. These early microbial profiles have also been shown to be associated with protection against any respiratory infection in later childhood $[9,14,20]$. The exact role of Moraxellaceae is still controversial. The Moraxellaceae family has been suggested to be a symbiotic stabilizer of the nasal microbiome in early life and associated with a lower probability of developing upper respiratory tract infections $[11,12]$. However, this observation has not been consistent between studies [14, $21,22]$. 


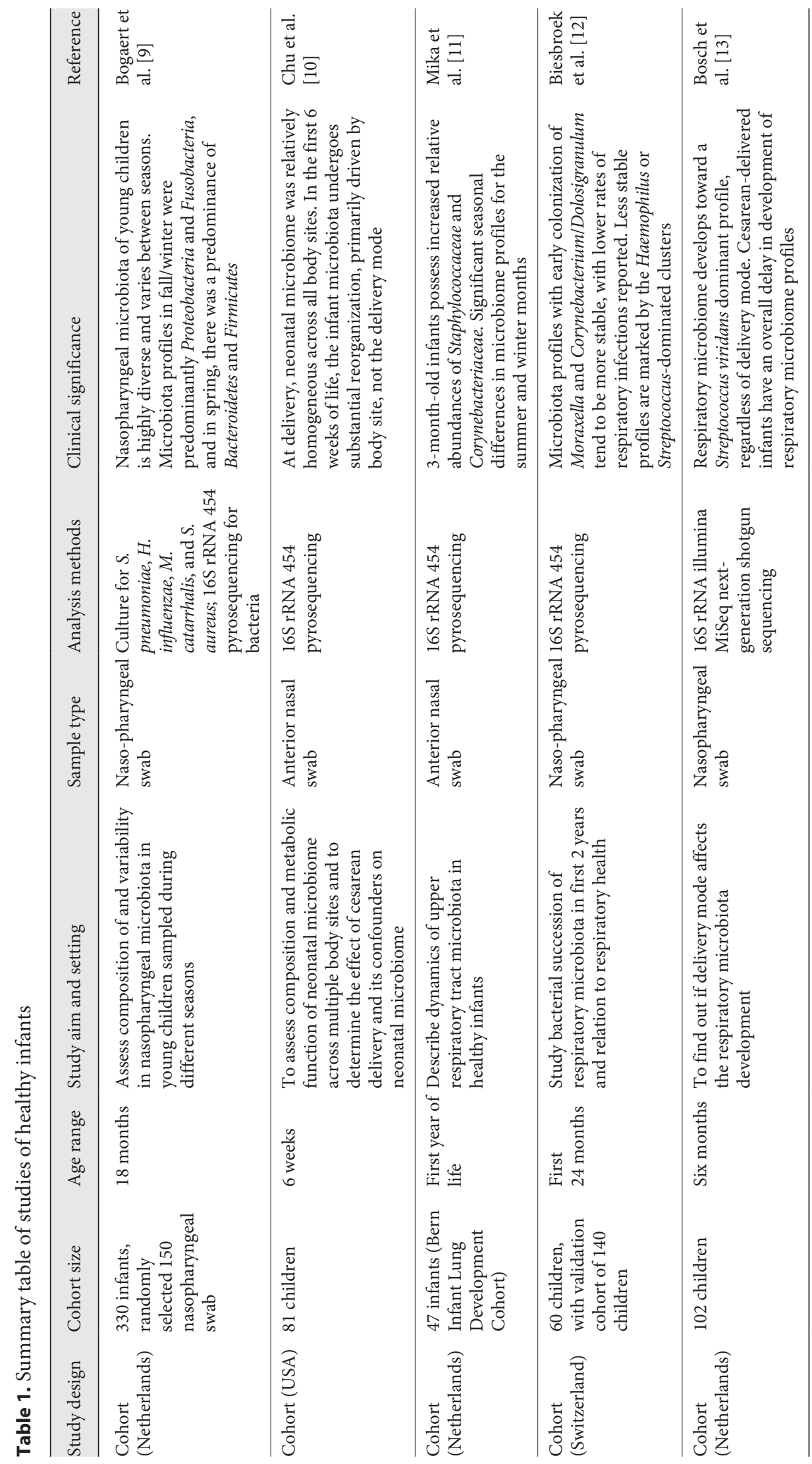


Fig. 1. Graphic representation of early establishment and succession of nasal microbiota (represented as relative abundance) in healthy infants in the first 2 years of life and adolescent/adult (not drawn to actual scale). Nasopharyngeal microbiome of healthy infants is dominated by a high abundance of Corynebacteriaceae, Moraxellaceae, and Staphylococcaceae in the first few months of life before subsequently replaced by a dominance of Streptococcaceae and/or either Corynebacteriaceae or $\mathrm{Mo}$ raxellaceae and other minor families.

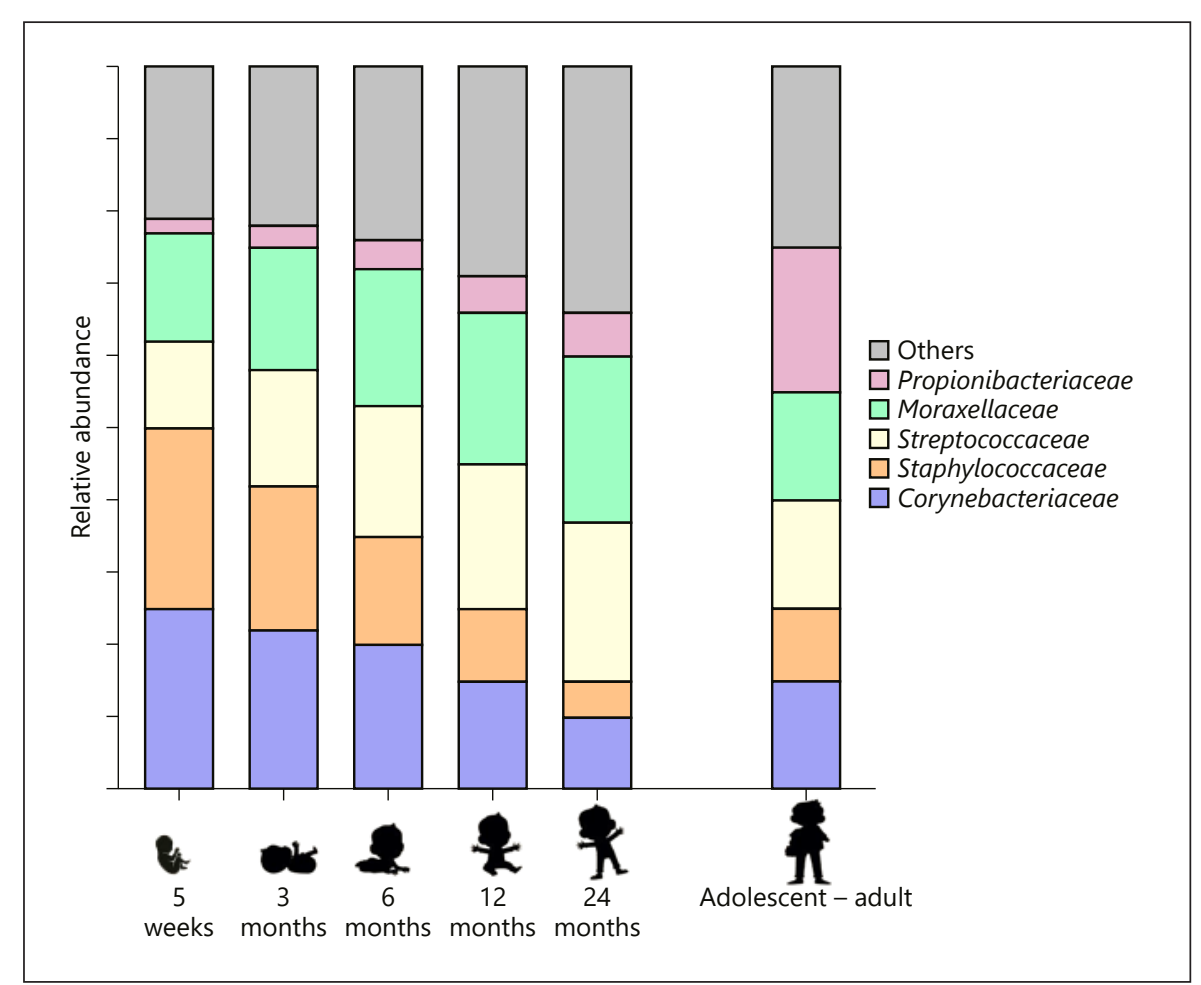

Bacteria with low abundance may also play a role in a healthy infant's respiratory tract. Lactobacillus spp. is a probiotic bacterium that is well known for its protective effects on the human gut microbiome and gut health. In recent years, longitudinal profiles of the healthy infants' nasal microbiome have shown that although Lactobacillus is a minor bacteria ( $<1 \%$ relative abundance), it could provide protection from infection and disorders, likely by inhibiting pathogenic bacteria growth through inhibitory substances such as bacteriocins $[9,10]$.

\section{Infant Nasal Microbiota and the Development of Rhinitis and Wheeze Disorders in Childhood}

Compared to healthy infants, a reduced diversity of nasal microbiota is associated with subsequent rhinitis and wheezing disorders, as well as allergen sensitization $[19,23,24]$. These studies suggest that colonization with a diverse array of microbes may be protective against inflammatory disorders of the upper and lower airways. The nasal microbiota promotes host resistance against infection with pathogens by (1) competing for sites of colonization and (2) direct production of inhibitory molecules and depletion of nutrients to prevent proliferation of pathogens. Table 2 summarizes 11 studies performed in both birth cohorts and cross-sectional studies which provide insights into the association between the infant nasal bacterial/viral microbiota succession/profiles. Their contribution to the subsequent development of respiratory disorders such as wheeze and rhinitis in childhood is also depicted in Figure 2.

There is evidence that Corynebacterium spp. is an important commensal for the development of healthy infant nasal microbiota. The Growing Up in Singapore toward Healthy Outcomes (GUSTO) birth cohort study showed that the overall abundance of Corynebacterium spp. found in the nasal microbiota was enriched in healthy infants compared to infants with rhinitis and/or wheeze in the first 18 months of life [19]. Moreover, Corynebacterium spp. in nasal epithelium of healthy infants has been shown to inhibit $S$. aureus colonization, which in turn reduced S. aureus infections [24]. Biesbroek and colleagues [12] also reported a lower incidence of upper respiratory tract infections in infants with a Corynebacterium-dominated nasal microbiome profile in early life.

Apart from Corynebacterium spp., a high abundance of $S$. epidermidis is also associated with a reduced presence of potential pathogens such as $S$. aureus and $M$. catarrhalis. S. epidermidis was shown to exhibit high anti- 


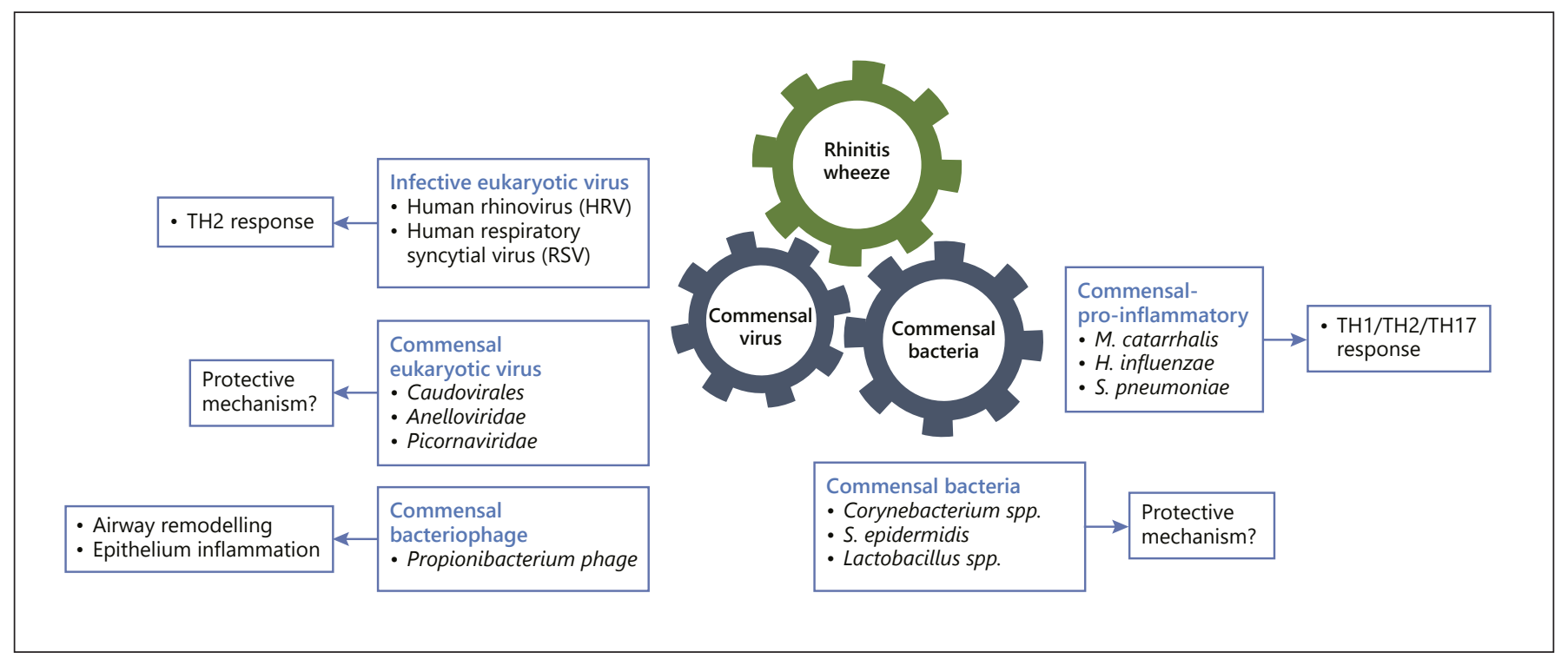

Fig. 2. Graphic representation of the potential role of upper airway bacteria, eukaryotic viruses, and bacteriophages in the pathogenesis of rhinitis and/or wheezing.

microbial peptide-inducing and biofilm-forming capacities which help to outcompete pathogenic bacteria during nasal colonization and has the ability to protect itself from epithelial antimicrobial peptides in vivo [25]. In addition, Rosas-Salazar et al. [26] found a lower abundance of Lactobacillus in the nasal microbiome of wheezing infants in the Infant Susceptibility to Pulmonary Infections and Asthma Following RSV Exposure (INSPIRE) longitudinal cohort. It could thus be postulated that the presence of Lactobacillus might be protective against development of viral-induced wheezing disorders.

On the other hand, early nasal establishment with proinflammatory bacteria species has been postulated to predispose infants to respiratory disorders such as rhinitis and wheezing. The Proteobacteria phylum, predominantly Oxalobacteraceae and Moraxellaceae genera, was found to be more abundant in infants with rhinitis and concomitant wheeze in the first 18 months of life (Singapore) and in dust mite-sensitized rhinitis and asthma infant at 3-5 years of age (Taiwan), respectively $[19,23]$.

In a culture-based study, Bisgaard and colleagues [27] observed an association between increased abundance of M. catarrhalis and H. influenzae (Proteobacteria phylum) and S. pneumoniae (Firmicutes phylum) in hypopharyngeal secretions of asymptomatic 1-month-old infants, and subsequent acute wheeze. Furthermore, Teo and colleagues [14] demonstrated with 16S rRNA sequencing that higher abundance of Streptococcus spp. in the nasal microbiome of 7-9-week-old infants was associated with an increased prevalence for chronic wheeze by 5 years of age. These earlier reports are consistent with findings of a Danish cohort of children born to mothers with asthma. The authors also found an association between colonization with $H$. influenzae, S. pneumoniae, or M. catarrhalis in the upper airways at 1 month and development of troublesome lung symptoms such as cough and wheezing within first year of life [28]. A more recent study involving the COAST birth cohort study reported that overrepresentation of these same bacteria, $H$. influenzae, $S$. pneumoniae, or M. catarrhalis, during wheezing illnesses in the first 2 years of life was associated with asthma in later childhood. Moreover, a Staphylococcus-dominant microbiome (predominance of $S$. aureus) in the first 6 months of life was associated with increased risk of recurrent wheezing by age 3 years [29]. In another study in Ecuadorian infants with noninfectious early-onset wheezing, there was a significantly higher abundance of Neisseriaceae and Haemophilus families in wheezing children at 10 months, compared to healthy subjects [30]. These longitudinal studies strongly support the notion that the early establishment of certain bacteria belonging to the Proteobacteria and Firmicutes phyla could predispose to subsequent development of rhinitis and wheeze disorders in early childhood. 


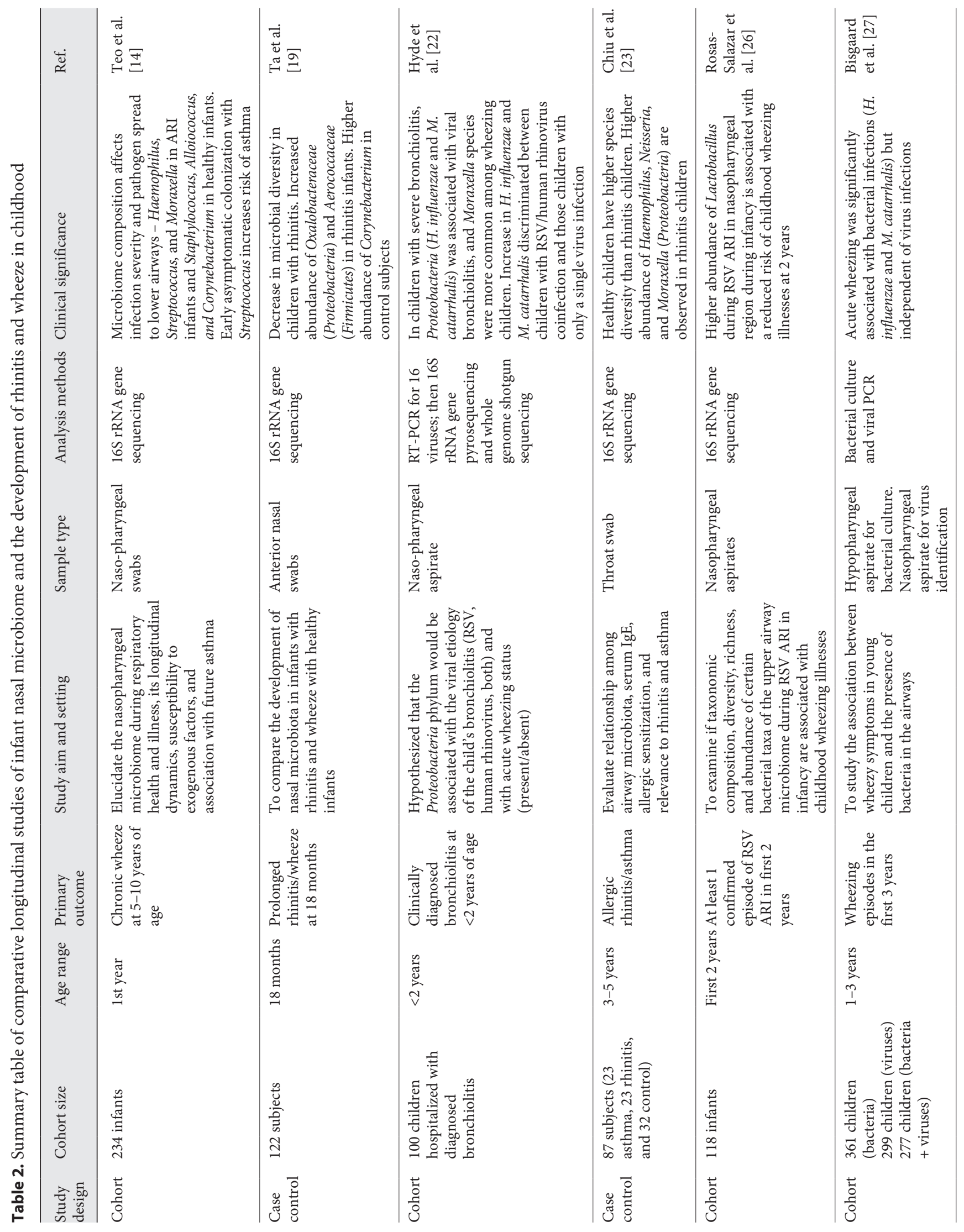




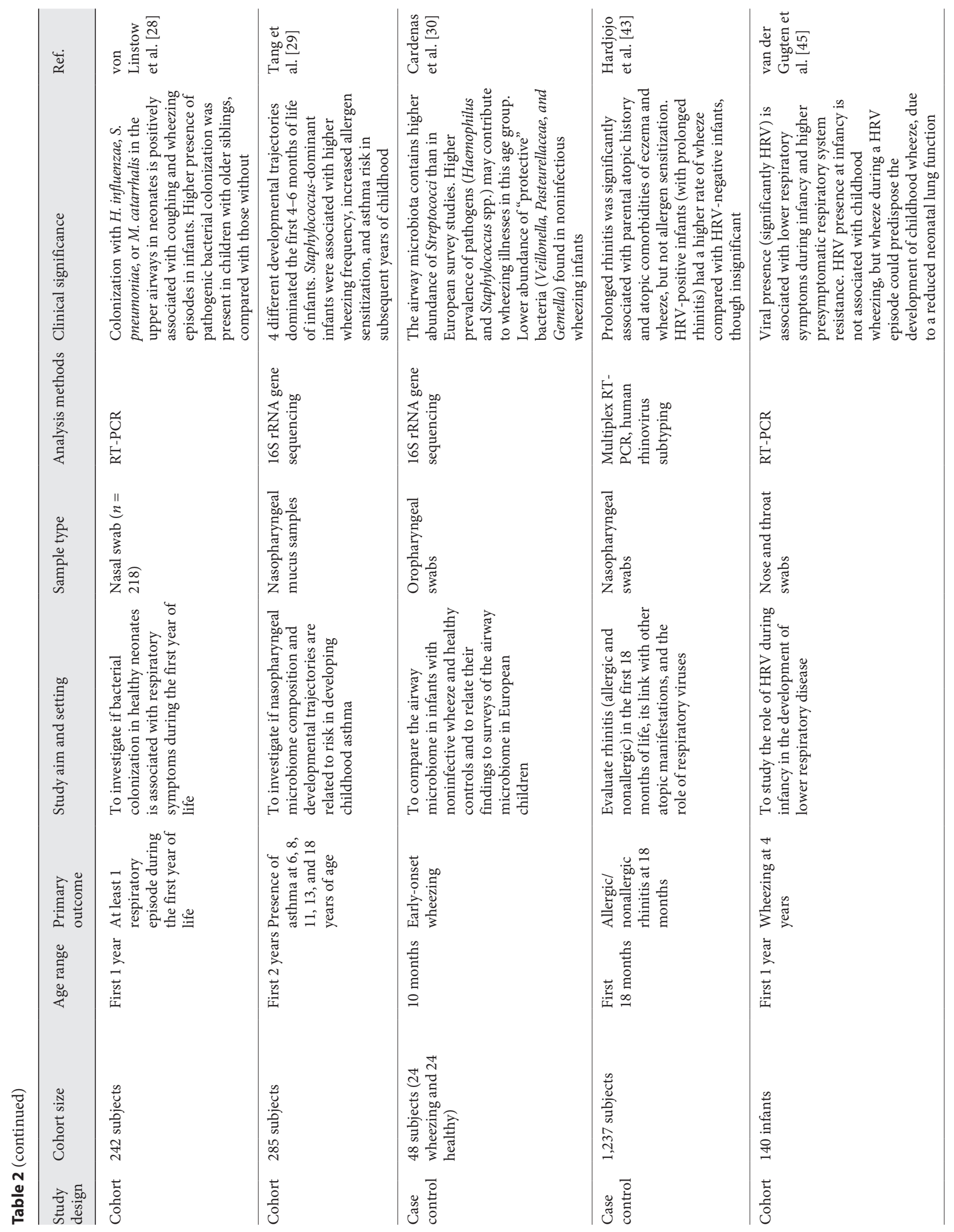




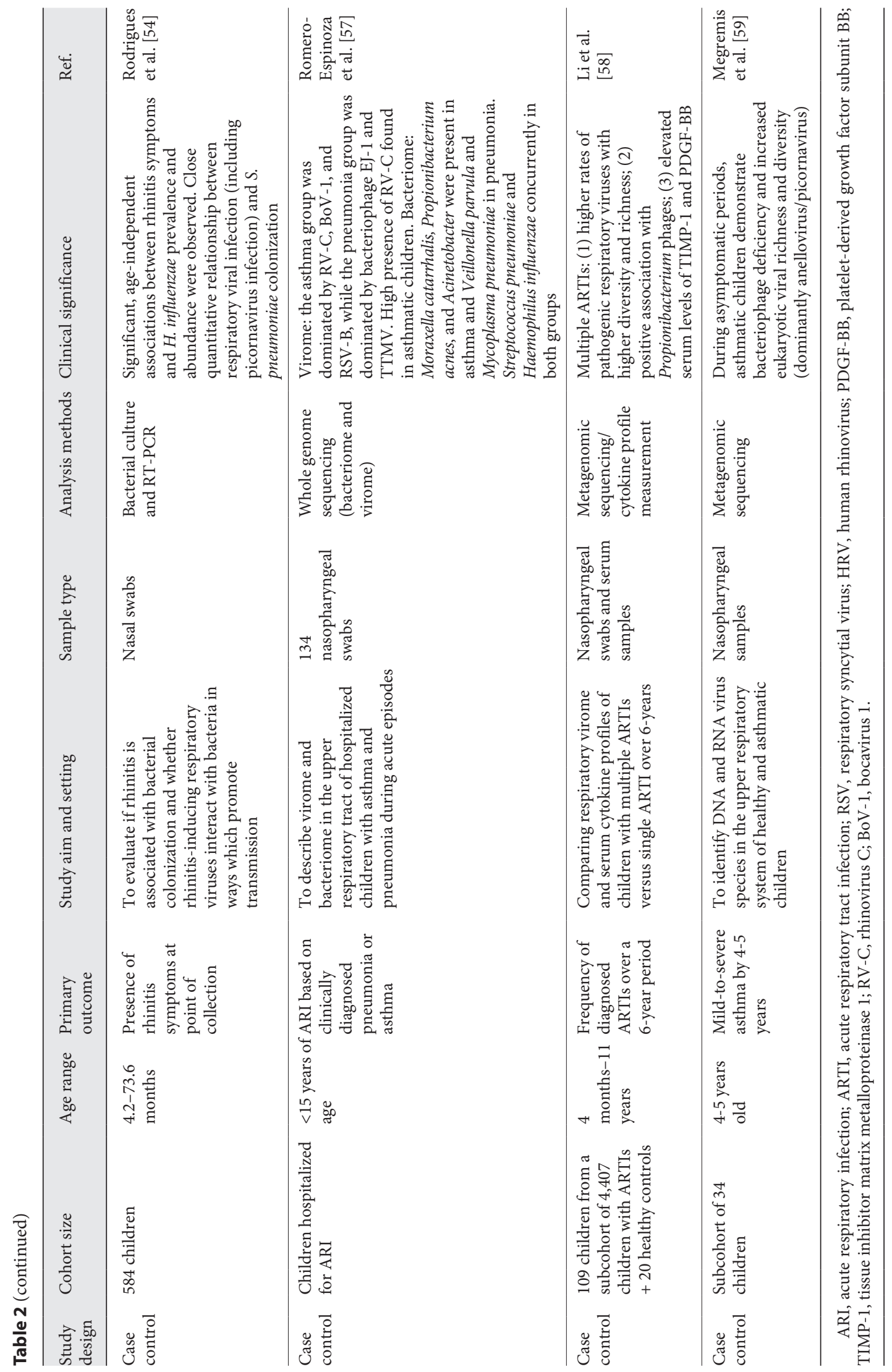


Proinflammatory Commensals and the Development of Childhood Respiratory Disorders

Several mechanisms have been proposed to explain the links between early proinflammatory bacterial colonization, in particular S. pneumoniae, H. influenzae, and M. catarrhalis, and the development of respiratory disorders. The survival of these commensals in the nasal mucosa has been attributed to their ability to form interspecies biofilms. Biofilm formation between $S$. pneumoniae and $H$. influenzae confers increased resistance to host antimicrobial agents (lysozyme, lactoferrin, and lipocalin), increased expression of cell surface appendages (type IV pili, lipopolysaccharide [LPS], or lipooligosaccharide) [31, 32], and the release of biomolecules such as extracellular DNA $[33,34]$, which further promotes the adhesion and cohesion properties of biofilm cells. Moreover, studies also showed that co-colonization with $M$. catarrhalis whose outer membrane vesicles contain phospholipids, adhesins, and immunomodulatory compounds, such as lipooligosaccharide, increases resistance of biofilms to antibiotics (amoxicillin) and host clearance [35]. Autoinducer-2 - an essential quorum signaling molecule for interbacterial communication - which is conserved among numerous bacterial species including $H$. influenzae enhances the establishment and maturation of polymicrobial biofilms containing these species [36].

Furthermore, colonization by these proinflammatory bacteria induces species-specific stimulation of the immune responses of the airway mucosa and increases inflammation accompanied by higher production and release of host antimicrobial peptides and proteins in the nasal epithelium via activation of Toll-like receptors (TLR). Lipoteichoic acids of S. pneumoniae are sensed by TLR-2 [37], whereas lipoproteins and LPS of $H$. influenzae are recognized by TLR2 and TLR-4, respectively [38]; TLR- 4 has also been reported to recognize the pore-forming toxin pneumolysin of $S$. pneumoniae [39]. To substantiate these in vitro findings, the Copenhagen Prospective Study of Asthma in Childhood 2010 birth cohort demonstrated that the upper airway lining fluid of neonates colonized with $M$. catarrhalis, $H$. influenzae, and S. pneumoniae had higher levels of innate/ TH1/TH2/TH17-related cytokines and chemokines compared to noncolonized neonates [40]. Infants who had proinflammatory responses induced by these bacteria were subsequently shown to be at higher risk of asthma at the age of 7 years. The investigators also showed that peripheral blood mononuclear cells collected from these children at age 6 months induced aberrant production of TH2 and TH17 cytokines in response to $H$ influenza, $M$ catarrhalis, and $S$ pneumoniae compared to nonasthma controls [41].
These studies suggest that early colonization with these proinflammatory bacteria could influence type 2 chronic inflammation and subsequent asthma.

\section{Nasal Virome}

The respiratory virome is also an integral component of the nasal microbiome, encompassing eukaryotic and prokaryotic (bacteriophages) viruses which are found in the upper respiratory tract and within the lung. However, little is known about the role of bacteriophages and their associations with eukaryotic viruses in respiratory health. Moreover, there is still a lack of mechanistic data to provide insights into the physiological role of commensal viruses in respiratory homeostasis.

The role of infectious eukaryotic viruses during episodes of acute respiratory tract infections (ARTIs) in young children and their impact on subsequent development of wheezing disorders are well documented. Human rhinovirus (HRV) and human respiratory syncytial virus (RSV) are major viral pathogens that trigger rhinitis and wheezing disorders in infants and are also risk factors for subsequent recurrent wheeze disorders in later childhood [27, 42-45]. These viruses incite lung damage through various immunological pathways such as the activation of proinflammatory mediators (such as TNF- $\alpha$, interleukins, and chemokines) as well as activation of leukocytes at the infection site, resulting in lung function impairment, persistent bronchial hyperreactivity, airway inflammation, and promoting Th2 sensitization [46]. Moreover, children with concomitant allergen sensitization and skewed Th2-mediated responses were shown to have impaired type I and type III interferons leading to increased susceptibility to RSV and rhinovirus infections and recurrent wheezing $[47,48]$.

Respiratory viruses such as HRV and RSV can trigger an outgrowth, rather than a new acquisition of S. pneumoniae and $M$. catarrhalis from the resident microbial community [49], as well as enhance their adherence to the respiratory epithelium by upregulating the expression of bacterial adhesion molecules such as fibronectin and platelet-activating factor receptor [50], which in turn facilitate secondary bacterial infection [51], and depress innate immune responses such as neutrophil and natural killer cell recruitment [52]. HRV was also shown to induce impairment on epithelial proliferation and promote airway remodeling [53].

Far less is known about the commensal virome, particularly its succession from birth, and its role in modulating respiratory health. Several technical challenges exist in studying the nasal virome. The low biomass and subopti- 
mal viral nucleic acid extraction technology currently available limits viral detection capabilities. The odds of detecting viral agents in a study of infants and young children up to 5 years old decreased significantly (up to $70 \%$ ) with increasing age [54], which might be due to fewer viral infections in older children. For these reasons, untargeted metagenomic sequencing for the identification of commensal viruses in nasal specimens has not been systematically evaluated. Furthermore, knowledge of bacteriophages has lagged substantially behind that of bacteria and other members of the microbiome due to technical difficulties in the isolation of bacteriophages and limited reference databases for phage genomes [55]. Nevertheless, the improvement of molecular detection techniques, including standardization, is already and will continue to facilitate the field [56].

A recent metagenomic sequencing study of the nasal virome in hospitalized asthmatic children below 15 years of age revealed that rhinovirus $\mathrm{C}$, bocavirus 1 , RSV-B, and parvovirus B19 were commonly detected, and bacteriophages such as Propionibacterium phage, Staphylococcus phage, and Streptococcus phage were also present, albeit with lower read counts [57]. Another study reported a significant elevation of relative abundance of Propionibacterium phages in children with multiple ARTIs compared to those with isolated ARTIs. This relative increase in abundance of these phages was associated with higher levels of serum tissue inhibitor matrix metalloproteinase 1 and platelet-derived growth factor subunit BB, which are involved in airway remodeling and epithelial inflammation conferring susceptibility to common respiratory viral infections or colonization by pathogenic bacteria [58]. These studies shed light on the possible role of bacteriophages on the upper respiratory tract and lung infections in children.

To the best of our knowledge, there is only 1 preprint study evaluating the nasal virome in asymptomatic children [59]. This study evaluated the nasal virome in European preschool children with stable asthma and who were symptom-free for at least 1 month. They showed a relative bacteriophage deficiency (richness and diversity) and increased eukaryotic viral presence, predominantly Anelloviruses, compared to healthy controls, indicating that children with asthma have a characteristically dysbiotic virome. The core eukaryotic virome in both groups consisted of Caudovirales, Anelloviridae, and Picornaviridae families. However, as this study was conducted in children with known asthma, it is not known if the differences observed in the respiratory virome during stable asthma episodes might be attributed to the disease itself or its treatment. Despite its small sample size, this study suggests that maintaining a stable respiratory core virome comprising eukaryote viruses and bacteriophages could be functionally important for the maintenance of a "healthy" equilibrium and could confer protection against asthma symptoms. Bacteriophages have been suggested to play an essential role in regulating bacterial populations within the respiratory tract that would in turn prime and modulate host immune responses in children [60-62].

\section{Conclusion}

This review provides a broad overview of the current body of evidence relating to the human upper respiratory microbiota and virome and its influence on rhinitis and wheezing disorders in children. There are substantial data showing that there are distinct differences in nasal microbiota composition in children with respiratory disorders and that colonization patterns in early life appear to impact development of disease in later life. There is also now emerging evidence that commensal respiratory virome, including bacteriophages, also play a role in childhood respiratory infections and asthma, but advances in viral detection technology are needed to improve our understanding of the hostvirome interactions in disease pathogenesis. Understanding the influence of the commensal microbiota and virome on the integrity of the respiratory tract and mucosal immune responses is an important step toward the implementation of strategies to reduce susceptibility to these disorders.

\section{Conflict of Interest Statement}

The authors have no conflicts of interest to declare.

\section{Funding Sources}

Tham E.H. was supported by the National Medical Research Council (NMRC) Research Training Fellowship grant ( $\mathrm{MH}$ 095:003\008-225) and the Transition Award (MOH-000269) from NMRC, Singapore. Lee B.W. was supported by the Clinician-Scientist Individual Research Grant (NMRC/CIRG/1344/2012) from NMRC, Singapore.

\section{Author Contributions}

The named authors meet the International Committee of Medical Journal Editors (ICMJE) criteria for authorship of this paper. C.J.X. Tay, L.D.H. Ta, Y.X.O. Yeong, and G.C. Yap prepared the manuscript. J.J.H. Chu, B.W. Lee, and E.H. Tham reviewed this paper. All authors reviewed and approved the final draft for publication. 


\section{References}

1 Turnbaugh PJ, Ley RE, Hamady M, FraserLiggett CM, Knight R, Gordon JI. The human microbiome project. Nature. 2007;449(7164): 804-10.

2 Scholtens PA, Oozeer R, Martin R, Amor KB, Knol J. The early settlers: intestinal microbiology in early life. Annu Rev Food Sci Technol. 2012;3:425-47.

3 Man WH, de Steenhuijsen Piters WA, Bogaert $\mathrm{D}$. The microbiota of the respiratory tract: gatekeeper to respiratory health. Nat Rev Microbiol. 2017;15(5):259-70.

4 Charlson ES, Bittinger K, Haas AR, Fitzgerald AS, Frank I, Yadav A, et al. Topographical continuity of bacterial populations in the healthy human respiratory tract. Am J Respir Crit Care Med. 2011;184(8):957-63.

5 Laufer AS, Metlay JP, Gent JF, Fennie KP, Kong Y, Pettigrew MM. Microbial communities of the upper respiratory tract and otitis media in children. mBio. 2011;2(1):e00245-10.

6 Chen CH, Liou ML, Lee CY, Chang MC, Kuo HY, Chang TH. Diversity of nasal microbiota and its interaction with surface microbiota among residents in healthcare institutes. Sci Rep. 2019;9(1):6175.

7 Rawls M, Ellis AK. The microbiome of the nose. Ann Allergy Asthma Immunol. 2019; 122(1):17-24.

8 Sakwinska O, Bastic Schmid V, Berger B, Bruttin A, Keitel K, Lepage M, et al. Nasopharyngeal microbiota in healthy children and pneumonia patients. J Clin Microbiol. 2014; 52(5):1590-4.

9 Bogaert D, Keijser B, Huse S, Rossen J, Veenhoven R, van Gils E, et al. Variability and diversity of nasopharyngeal microbiota in children: a metagenomic analysis. PLoS One. 2011;6(2):e17035

10 Chu DM, Ma J, Prince AL, Antony KM, Seferovic MD, Aagaard KM. Maturation of the infant microbiome community structure and function across multiple body sites and in relation to mode of delivery. Nat Med. 2017; 23(3):314-26

11 Mika M, Mack I, Korten I, Qi W, Aebi S, Frey $\mathrm{U}$, et al. Dynamics of the nasal microbiota in infancy: a prospective cohort study. J Allergy Clin Immunol. 2015;135(4):905-e11.

12 Biesbroek G, Tsivtsivadze E, Sanders EA, Montijn R, Veenhoven RH, Keijser BJ, et al. Early respiratory microbiota composition determines bacterial succession patterns and respiratory health in children. Am J Respir Crit Care Med. 2014;190(11):1283-92.

13 Bosch AATM, Levin E, van Houten MA, Hasrat R, Kalkman G, Biesbroek G, et al. Development of upper respiratory tract microbiota in infancy is affected by mode of delivery. EBioMedicine. 2016;9:336-45.

14 Teo SM, Mok D, Pham K, Kusel M, Serralha $\mathrm{M}$, Troy N, et al. The infant nasopharyngeal microbiome impacts severity of lower respiratory infection and risk of asthma development. Cell Host Microbe. 2015;17(5):704-15.
15 Bosch AATM, de Steenhuijsen Piters WAA, van Houten MA, Chu MLJN, Biesbroek G, Kool J, et al. Maturation of the infant respiratory microbiota, environmental drivers, and health consequences. A prospective cohort study. Am J Respir Crit Care Med. 2017; 196(12):1582-90.

16 Bomar L, Brugger SD, Lemon KP. Bacterial microbiota of the nasal passages across the span of human life. Curr Opin Microbiol. 2018;41:8-14.

17 Human Microbiome Project C. Structure, function and diversity of the healthy human microbiome. Nature. 2012;486(7402):20714.

18 Oh J, Conlan S, Polley EC, Segre JA, Kong $\mathrm{HH}$. Shifts in human skin and nares microbiota of healthy children and adults. Genome Med. 2012;4(10):77.

19 Ta LDH, Yap GC, Tay CJX, Lim ASM, Huang $\mathrm{CH}$, Chu CW, et al. Establishment of the nasal microbiota in the first 18 months of life: correlation with early-onset rhinitis and wheezing. J Allergy Clin Immunol. 2018;142(1):8695.

20 Hilty M, Qi W, Brugger SD, Frei L, Agyeman P, Frey PM, et al. Nasopharyngeal microbiota in infants with acute otitis media. J Infect Dis. 2012;205(7):1048-55.

21 Depner M, Ege MJ, Cox MJ, Dwyer S, Walker AW, Birzele LT, et al. Bacterial microbiota of the upper respiratory tract and childhood asthma. J Allergy Clin Immunol. 2017;139(3): 826-e13.

22 Hyde ER, Petrosino JF, Piedra PA, Camargo CA Jr, Espinola JA, Mansbach JM. Nasopharyngeal Proteobacteria are associated with viral etiology and acute wheezing in children with severe bronchiolitis. J Allergy Clin Immunol. 2014;133(4):1220-2.

23 Chiu CY, Chan YL, Tsai YS, Chen SA, Wang CJ, Chen KF, et al. Airway microbial diversity is inversely associated with mite-sensitized rhinitis and asthma in early childhood. Sci Rep. 2017;7(1):1820.

24 Khamash DF, Mongodin EF, White JR, Voskertchian A, Hittle L, Colantuoni E, et al. The association between the developing nasal $\mathrm{mi}-$ crobiota of hospitalized neonates and Staphylococcus aureus colonization. Open Forum Infect Dis. 2019;6(4):ofz062.

25 Liu Q, Liu Q, Meng H, Lv H, Liu Y, Liu J, et al. Staphylococcus epidermidis contributes to healthy maturation of the nasal microbiome by stimulating antimicrobial peptide production. Cell Host Microbe. 2020;27(1):68-78.e5.

26 Rosas-Salazar C, Shilts MH, Tovchigrechko A, Schobel S, Chappell JD, Larkin EK, et al. Nasopharyngeal Lactobacillus is associated with a reduced risk of childhood wheezing illnesses following acute respiratory syncytial virus infection in infancy. J Allergy Clin Immunol. 2018;142(5):1447-56.e9.
27 Bisgaard H, Hermansen MN, Bonnelykke K, Stokholm J, Baty F, Skytt NL, et al. Association of bacteria and viruses with wheezy episodes in young children: prospective birth cohort study. BMJ. 2010;341:c4978.

28 von Linstow ML, Schonning K, Hoegh AM, Sevelsted A, Vissing NH, Bisgaard H. Neonatal airway colonization is associated with troublesome lung symptoms in infants. Am J Respir Crit Care Med. 2013;188(8):1041-2.

29 Tang HHF, Lang A, Teo SM, Judd LM, Gangnon R, Evans MD, et al. Developmental patterns in the nasopharyngeal microbiome during infancy are associated with asthma risk. J Allergy Clin Immunol. 2020.

30 Cardenas PA, Cooper PJ, Cox MJ, Chico M, Arias C, Moffatt MF, et al. Upper airways microbiota in antibiotic-naïve wheezing and healthy infants from the tropics of rural Ecuador. PLoS One. 2012;7(10):e46803.

31 Jones PA, Samuels NM, Phillips NJ, Munson RS Jr, Bozue JA, Arseneau JA, et al. Haemophilus influenzae type b strain A2 has multiple sialyltransferases involved in lipooligosaccharide sialylation. J Biol Chem. 2002;277(17): 14598-611.

32 Carruthers MD, Tracy EN, Dickson AC, Ganser KB, Munson RS Jr, Bakaletz LO. Biological roles of nontypeable Haemophilus influenzae type IV pilus proteins encoded by the pil and com operons. J Bacteriol. 2012;194(8):192733.

33 Romao S, Memmi G, Oggioni MR, Trombe MC. LuxS impacts on LytA-dependent autolysis and on competence in Streptococcus pneumoniae. Microbiology. 2006;152(Pt 2): 333-41.

34 Jurcisek JA, Bakaletz LO. Biofilms formed by nontypeable Haemophilus influenzae in vivo contain both double-stranded DNA and type IV pilin protein. J Bacteriol. 2007;189(10): 3868-75.

35 Schaar V, Nordstrom T, Morgelin M, Riesbeck K. Moraxella catarrhalis outer membrane vesicles carry beta-lactamase and promote survival of Streptococcus pneumoniae and Haemophilus influenzae by inactivating amoxicillin. Antimicrob Agents Chemother. 2011;55(8):3845-53.

36 Armbruster CE, Hong W, Pang B, Weimer $\mathrm{KE}$, Juneau RA, Turner J, et al. Indirect pathogenicity of Haemophilus influenzae and Moraxella catarrhalis in polymicrobial otitis media occurs via interspecies quorum signaling. mBio. 2010;1(3):e00102-10.

37 Gonzalez-Zorn B, Senna JP, Fiette L, Shorte S, Testard A, Chignard M, et al. Bacterial and host factors implicated in nasal carriage of methicillin-resistant Staphylococcus aureus in mice. Infect Immun. 2005;73(3):1847-51.

38 Zola TA, Lysenko ES, Weiser JN. Mucosal clearance of capsule-expressing bacteria requires both TLR and nucleotide-binding oligomerization domain 1 signaling. J Immunol. 2008;181(11):7909-16. 
39 Malley R, Henneke P, Morse SC, Cieslewicz MJ, Lipsitch M, Thompson CM, et al. Recognition of pneumolysin by Toll-like receptor 4 confers resistance to pneumococcal infection. Proc Natl Acad Sci U S A. 2003;100(4):196671.

40 Folsgaard NV, Schjorring S, Chawes BL, Rasmussen MA, Krogfelt KA, Brix S, et al. Pathogenic bacteria colonizing the airways in asymptomatic neonates stimulates topical in flammatory mediator release. Am J Respir Crit Care Med. 2013;187(6):589-95.

41 Larsen JM, Brix S, Thysen AH, Birch S, Rasmussen MA, Bisgaard $\mathrm{H}$. Children with asthma by school age display aberrant immune responses to pathogenic airway bacteria as infants. J Allergy Clin Immunol. 2014;133(4): 1008-13.

42 Kusel MM, de Klerk NH, Kebadze T, Vohma V, Holt PG, Johnston SL, et al. Early-life respiratory viral infections, atopic sensitization, and risk of subsequent development of persistent asthma. J Allergy Clin Immunol. 2007; 119(5):1105-10.

43 Hardjojo A, Goh A, Shek LP, Van Bever HP Teoh OH, Soh JY, et al. Rhinitis in the first 18 months of life: exploring the role of respiratory viruses. Pediatr Allergy Immunol. 2015; 26(1):25-33.

44 Jackson DJ, Gangnon RE, Evans MD, Roberg KA, Anderson EL, Pappas TE, et al. Wheezing rhinovirus illnesses in early life predict asthma development in high-risk children. Am J Respir Crit Care Med. 2008;178(7):667-72.

45 van der Gugten AC, van der Zalm MM, Uiterwaal CS, Wilbrink B, Rossen JW, van der Ent CK. Human rhinovirus and wheezing: short and long-term associations in children. Pediatr Infect Dis J. 2013;32(8):827-33.

46 Rossi GA, Colin AA. Infantile respiratory syncytial virus and human rhinovirus infections: respective role in inception and persis- tence of wheezing. Eur Respir J. 2015;45(3): 774-89.

47 Xatzipsalti M, Psarros F, Konstantinou G, Gaga M, Gourgiotis D, Saxoni-Papageorgiou $\mathrm{P}$, et al. Modulation of the epithelial inflammatory response to rhinovirus in an atopic environment. Clin Exp Allergy. 2008;38(3): $466-72$.

48 Edwards MR, Strong K, Cameron A, Walton RP, Jackson DJ, Johnston SL. Viral infections in allergy and immunology: how allergic inflammation influences viral infections and illness. J Allergy Clin Immunol. 2017;140(4): 909-20.

49 Brealey JC, Young PR, Sloots TP, Ware RS, Lambert SB, Sly PD, et al. Bacterial colonization dynamics associated with respiratory syncytial virus during early childhood. Pediatr Pulmonol. 2020;55(5):1237-45.

50 Wang JH, Kwon HJ, Jang YJ. Rhinovirus enhances various bacterial adhesions to nasal epithelial cells simultaneously. Laryngoscope. 2009;119(7):1406-11.

51 Mallia P, Footitt J, Sotero R, Jepson A, Contoli M, Trujillo-Torralbo MB, et al. Rhinovirus infection induces degradation of antimicrobial peptides and secondary bacterial infection in chronic obstructive pulmonary disease. Am J Respir Crit Care Med. 2012; 186(11):1117-24

52 Robinson KM, Lee B, Scheller EV, Mandalapu S, Enelow RI, Kolls JK, et al. The role of IL-27 in susceptibility to post-influenza Staphylococcus aureus pneumonia. Respir Res. 2015; 16:10.

53 Bossios A, Psarras S, Gourgiotis D, Skevaki CL, Constantopoulos AG, Saxoni-Papageorgiou $\mathrm{P}$, et al. Rhinovirus infection induces cytotoxicity and delays wound healing in bronchial epithelial cells. Respir Res. 2005;6:114.

54 Rodrigues F, Foster D, Nicoli E, Trotter C, Vipond $\mathrm{B}$, Muir $\mathrm{P}$, et al. Relationships between rhinitis symptoms, respiratory viral infections and nasopharyngeal colonization with Streptococcus pneumoniae, Haemophilus influenzae and Staphylococcus aureus in children attending daycare. Pediatr Infect Dis J. 2013;32(3):227-32

55 Klumpp J, Fouts DE, Sozhamannan S. Next generation sequencing technologies and the changing landscape of phage genomics. Bacteriophage. 2012;2(3):190-9.

56 Rosenthal LA, Avila PC, Heymann PW, Martin RJ, Miller EK, Papadopoulos NG, et al. Viral respiratory tract infections and asthma: the course ahead. J Allergy Clin Immunol. 2010;125(6):1212-7.

57 Romero-Espinoza JA, Moreno-Valencia Y, Coronel-Tellez RH, Castillejos-Lopez M, Hernandez A, Dominguez A, et al. Virome and bacteriome characterization of children with pneumonia and asthma in Mexico City during winter seasons 2014 and 2015. PLoS One. 2018;13(2):e0192878.

58 Li Y, Fu X, Ma J, Zhang J, Hu Y, Dong W, et al. Altered respiratory virome and serum cytokine profile associated with recurrent respiratory tract infections in children. Nat Commun. 2019;10(1):2288.

59 Megremis S, Constantinides B, Xepapadaki P, Bachert C, Neurath-Finotto S, Jartti T, et al. Bacteriophage deficiency characterizes respiratory virome dysbiosis in childhood asthma. bioRxiv. 2020.

60 Mitchell AB, Oliver BG, Glanville AR. Translational aspects of the human respiratory virome. Am J Respir Crit Care Med. 2016; 194(12):1458-64

61 Abeles SR, Robles-Sikisaka R, Ly M, Lum AG, Salzman J, Boehm TK, et al. Human oral viruses are personal, persistent and genderconsistent. ISME J. 2014;8(9):1753-67.

62 Virgin HW, Wherry EJ, Ahmed R. Redefining chronic viral infection. Cell. 2009;138(1):30-50. 